\title{
BMJ Open Safety and efficacy of the predictive low glucose management system in the prevention of hypoglycaemia: protocol for randomised controlled home trial to evaluate the Suspend before low function
}

\author{
M B Abraham, ${ }^{1,2} \mathrm{~J}$ A Nicholas, ${ }^{3} \mathrm{~T}$ T Ly, ${ }^{1,2,3}$ H C Roby, ${ }^{3}$ N Paramalingam, ${ }^{1,3}$ \\ J Fairchild, ${ }^{4}$ B R King, ${ }^{5}$ G R Ambler, ${ }^{6}$ F Cameron, ${ }^{7}$ E A Davis, ${ }^{1,2,3}$ T W Jones ${ }^{1,2,3}$
}

To cite: Abraham MB, Nicholas JA, Ly TT, et al. Safety and efficacy of the predictive low glucose management system in the prevention of hypoglycaemia: protocol for randomised controlled home trial to evaluate the Suspend before low function. BMJ Open 2016:6: 011589.

doi:10.1136/bmjopen-2016011589

- Prepublication history for this paper is available online. To view these files please visit the journal online (http://dx.doi.org/10.1136/ bmjopen-2016-011589).

Received 19 February 2016 Revised 16 March 2016 Accepted 22 March 2016

CrossMark

For numbered affiliations see end of article.

Correspondence to Professor T W Jones; Tim.Jones@health.wa.gov.au

\section{ABSTRACT}

Introduction: Innovations with sensor-augmented pump therapy (SAPT) to reduce hypoglycaemia in patients with type 1 diabetes are an ongoing area of research. The predictive low glucose management (PLGM) system incorporates continuous glucose sensor data into an algorithm and suspends basal insulin before the occurrence of hypoglycaemia. The system was evaluated in in-clinic studies, and has informed the parameters of a larger home trial to study its efficacy and safety in real life.

Methods and analysis: The aim of this report is to describe the study design and outcome measures for the trial. This is a 6-month, multicentre, randomised controlled home trial to test the PLGM system in children and adolescents with type 1 diabetes. The system is available in the Medtronic MiniMed 640G pump as the 'Suspend before low' feature. Following a run-in period, participants are randomised to either the control arm with SAPT alone or the intervention arm with SAPT and Suspend before low. The primary aim of this study is to evaluate the time spent hypoglycaemic (sensor glucose $<3.5 \mathrm{mmol} / \mathrm{L}$ ) with and without the system. The secondary aims are to determine the number of hypoglycaemic events, the time spent hyperglycaemic, and to evaluate safety with ketosis and changes in glycated haemoglobin. The study also aims to assess the changes in counter-regulatory hormone responses to hypoglycaemia evaluated by a hyperinsulinaemic hypoglycaemic clamp in a subgroup of patients with impaired awareness. Validated questionnaires are used to measure the fear of hypoglycaemia and the impact on the quality of life to assess burden of the disease.

Ethics and dissemination: Ethics committee permissions were gained from respective Institutional Review boards. The findings of the study will provide high quality evidence of the ability of the system in the prevention of hypoglycaemia in real life.

\section{Strengths and limitations of this study}

This is the first randomised controlled home tria which will provide high quality evidence of the efficacy and safety of the predictive low glucose management system in the prevention of hypoglycaemia in real life situations.

- Apart from the glycaemic data, the 6-month duration of the study provides the ability to evaluate the impact of this technology on various psychosocial parameters in both children and their caregivers.

- The study will also determine the ability to use the system as a tool in restoration of hypoglycaemia awareness.

- The settings for the Suspend before low feature are constant for the entire duration of the study; however, these settings can be changed in real life.

- This study is a paediatric study and hence, one of the challenges will be in supporting and encouraging sensor use in the adolescent age group.

Trial registration number: ACTRN12614000510640, Pre-results.

\section{INTRODUCTION}

Hypoglycaemia imposes a considerable burden of disease on individuals and their families living with type 1 diabetes. ${ }^{1}$ Interventions designed to reduce and prevent hypoglycaemia are an important focus of research, especially those related to sensor-augmented pump therapy (SAPT) 
and glucose control algorithms. The Medtronic Low Glucose Suspend (LGS) system automatically suspends basal insulin delivery for up to $2 \mathrm{~h}$ in response to sensordetected hypoglycaemia and thereby reduces the duration of hypoglycaemia. ${ }^{2}{ }^{3}$ This function has reduced the incidence of moderate and severe episodes of hypoglycaemia in patients with impaired awareness of hypoglycaemia (IAH), ${ }^{4}$ and has been found to be safe with no rebound hyperglycaemia and ketosis. ${ }^{5}{ }^{6}$ While this system suspends insulin delivery when the patient is hypoglycaemic, the next step is to suspend insulin delivery before the patient is hypoglycaemic. The capacity to predict hypoglycaemia and suspend insulin delivery before hypoglycaemia occurs offers the additional advantage of preventing hypoglycaemia with further reduction in the actual time spent hypoglycaemic.

A significant proportion of children and adolescents with type 1 diabetes have IAH with defective symptomatic and counter-regulatory hormone responses to hypoglycaemia. In a previous study by our group, IAH was reported by $29 \%$ of the clinic population. ${ }^{7}$ Though loss of hypoglycaemia awareness may be reversed by meticulous avoidance of hypoglycaemia for 3 weeks, ${ }^{8}$ this may be difficult to accomplish in real life and especially challenging for children. As predictive algorithms are designed to prevent hypoglycaemia, there may be an improvement in counter-regulatory hormones and return of adrenergic symptoms.

The initial in-clinic studies evaluating the predictive algorithms demonstrated a reduction in nocturnal and day-time hypoglycaemia induced by increased basal rates. ${ }^{9} 10$ The only home studies conducted to date were of short duration and used an investigational device overnight with a laptop-based Kalman filter predictive model controlling insulin delivery by Medtronic Veo system to assess the effect of the system on nocturnal hypoglycaemia. ${ }^{11}{ }^{12}$ In the era of rapidly evolving technology, various models have been used in the development of newer predictive algorithms. The predictive low glucose management (PLGM) system uses a different predictive algorithm and was initially evaluated in our centre under standardised in-clinic conditions. The system was an investigational device and the predictive algorithm was incorporated into the BlackBerry Storm smartphone which controlled the insulin infusion in participants on the Medtronic Paradigm Veo insulin pump and Enlite glucose sensor with MiniLink REAL-Time transmitter. The system was tested with moderate-intensity exercise, excess subcutaneous insulin bolus, and increased overnight basal rates which are common triggers of hypoglycaemia. With each hypoglycaemic stimulus, participants were randomised to a control arm with SAPT alone and an intervention arm with PLGM. The system suspended basal insulin when the sensor glucose was predicted to be $4.4 \mathrm{mmol} / \mathrm{L}$ and found a reduction in the need for treatment of hypoglycaemia for all three settings in the intervention arm. ${ }^{13}$ PLGM was also tested under in-clinic conditions in the
PILGRIM study. There was reduction in hypoglycaemia following administration of insulin bolus administration to virtual participants and following exercise in real life participants. ${ }^{14}$

Guided by the results of the in-clinic studies, the home trial was designed to evaluate the efficacy of the PLGM system in free-living conditions. The PLGM system is available in the Medtronic MiniMed 640G pump (Medtronic MiniMed, Northridge, California, USA) as the 'Suspend before low' feature which automatically suspends basal insulin infusion when hypoglycaemia is predicted. This system is evaluated in the home trial. In contrast to the PLGM in-clinic study, the home trial is of a longer duration, evaluates spontaneous rather than induced hypoglycaemia, and uses sensor glucose values rather than plasma glucose values to quantify hypoglycaemia. Although this system is commercially available, there are as yet no randomised controlled home trials for evaluating its efficacy in real life situations. A recent study with the system used for 4 weeks has shown that it can help patients avoid hypoglycaemia and is acceptable to them. ${ }^{15}$ Our study is the first randomised controlled home trial testing the Suspend before low function using the Medtronic MiniMed 640G pump in free-living conditions. Apart from the glycaemic data, the 6-month duration of the study provides the ability to assess the impact of the system in children and their caregivers.

We hypothesise that the PLGM system will reduce the time spent hypoglycaemic, with time spent in hypoglycaemia reduced by at least $40 \%$ during 6 months of therapy with SAPT and Suspend before low versus SAPT alone in patients with type 1 diabetes. The system will also not result in an increase in hyperglycaemia or ketosis, and will not result in a deterioration of glycaemic control as compared to standard SAPT. We further hypothesise that PLGM will improve hypoglycaemia awareness by reducing hypoglycaemia and will have a positive impact on the quality of life and reduce the fear of hypoglycaemia as determined by participant/ parent questionnaires. Finally, the patient acceptability of the PLGM system will be no worse than their acceptability of standard SAPT.

\section{AIMS}

The primary objective of the study is to compare the average percentage of time spent hypoglycaemic (sensor glucose level $<3.5 \mathrm{mmol} / \mathrm{L}$ ) during six months of therapy with SAPT and Suspend before low versus SAPT alone. The secondary objectives are to compare events of hypoglycaemia, defined as $20 \mathrm{~min}$ or more with sensor glucose $<3.5 \mathrm{mmol} / \mathrm{L}$, during 6 months of therapy in both groups and to compare the average percentage of time spent hypoglycaemic (sensor glucose level $<3.0 \mathrm{mmol} / \mathrm{L}$ ), in target range (sensor glucose 3.5$10 \mathrm{mmol} / \mathrm{L}$ ), and hyperglycaemic (sensor glucose level $10-15 \mathrm{mmol} / \mathrm{L}$ and $>15 \mathrm{mmol} / \mathrm{L})$. The study will also 
Table 1 Inclusion and exclusion criteria for PLGM home trial

\begin{tabular}{ll}
\hline Inclusion criteria & Exclusion criteria \\
\hline Age: 8-20 years & $\begin{array}{l}\text { Medical conditions predisposing } \\
\text { to hypoglycaemia other than } \\
\text { diabetes }\end{array}$ \\
$\begin{array}{l}\text { Oral glycaemic medications, eg } \\
\text { diabetes } \geq 1 \text { year }\end{array}$ & $\begin{array}{l}\text { metformin, sulphonylureas } \\
\text { Inability or refusal to meet } \\
\text { On CSII } \geq 6 \text { months }\end{array}$ \\
$\begin{array}{l}\text { HbA1c at eligibility } \\
\text { Pregol requirements }\end{array}$ \\
\hline $\begin{array}{l}\text { CSII, continuous subcutaneous insulin infusion CSII continuous } \\
\text { subcutaneous insulin infusion; HbA1c, glycated haemoglobin; }\end{array}$ \\
PLGM, predictive low glucose management.
\end{tabular}

evaluate the time spent hypoglycaemic during the day and night. In addition, we aim to determine the safety of the system by determining the number of ketosis events (blood ketones $>0.6 \mathrm{mmol} / \mathrm{L}$ ) and assess glycaemic control as measured by glycated haemoglobin (HbA1c) at the end of 6 months. The study will also determine the counter-regulatory hormone responses to hypoglycaemia that will be evaluated during a hyperinsulinaemic hypoglycaemic clamp study in a subgroup of participants with IAH. Hypoglycaemia awareness and the impact of diabetes on the patient's quality of life, fear of hypoglycaemia, patient satisfaction, and acceptability of the system will be evaluated using validated questionnaires administered at baseline, 3 months and end of the study.

The outcome or end point measures are based on the sensor glucose levels as predefined above. The other measures include the counter-regulatory hormone responses and adrenergic symptoms during hyperinsulinaemic hypoglycaemic clamp study, number of ketosis events defined as blood ketone $>0.6 \mathrm{mmol} / \mathrm{L}$, the incidence of moderate and severe hypoglycaemia, HbAlc, and the questionnaire scores at baseline, 3 months and 6 months of the study. Here, we provide methodological details of the PLGM home trial.

\section{METHODS}

This is a multicentre, unblinded, parallel, randomised controlled phase 3 home trial designed and conducted by five tertiary paediatric diabetes centres in Australia. The trial has been approved by Princess Margaret Hospital, Perth (HREC/2013121EP); The Children's Hospital at Westmead, Sydney (HREC/13/SCHN/405); John Hunter Children's Hospital, Newcastle (HREC/13/HNE/506); Royal Children's Hospital, Melbourne (HREC/13/HME/ 506); and Women's and Children's Hospital, Adelaide (HREC/13/WCHN/172). The trial is prospectively registered with the Australian New Zealand Clinical Trials Registry (ACTRN12614000510640). Inclusion and exclusion criteria are summarised in table 1.
As this is a new system evaluating safety of insulin delivery, we aim to include older children and adolescents with type 1 diabetes who are on continuous subcutaneous insulin infusion therapy (CSII). We recruit patients with $\mathrm{HbA} 1 \mathrm{c}<10 \%$ to reflect a clinic cohort and to exclude participants who are less likely to comply and adhere to the protocol. Participants are approached through the diabetes clinics and are screened for eligibility in the study. Written informed consent is obtained from participants aged $\geq 18$ years, and written parental consent and participant assent for those $<18$ years of age. Consent is attained by the research nurse or the doctor who is not directly involved in the routine care of the patient and their families. A participant may decide to withdraw from the study at any time without prejudice to their future care. The study duration for each participant is 6 months from randomisation. Based on previous unpublished data in a similar cohort of children and adolescents conducted in our centre, the control group is expected to spend on average $5.79 \%$ of the time with glucose level $<3.5 \mathrm{mmol} / \mathrm{L}$, with a SD of $4.87 \%$. 71 participants would be required in each group to have $80 \%$ power to detect a decrease of $2.3 \%$ (40\% reduction or effect size of 0.475 ) in the time spent with glucose level $<3.5 \mathrm{mmol} / \mathrm{L}$. It is anticipated that 175 participants would be recruited; based on the estimated dropout rate of $20 \%$, this will ensure a total of 142 participants for the duration of the trial, with at least 71 participants in each arm. Recruitment will cease once this target is achieved. The study started in August 2014 and is currently ongoing.

\section{PLGM system}

The predictive algorithm is available in the commercially available Medtronic MiniMed 640G pump (Medtronic MiniMed, Northridge, California, USA) that is designed for CSII integrated with real-time continuous glucose monitoring (CGM). The system consists of the Medtronic MiniMed 640G pump, CONTOUR NEXT LINK wireless blood glucose metre, Enlite glucose sensor and Guardian 2 Link transmitter. The transmitter sends the sensor glucose data wirelessly every $5 \mathrm{~min}$ to the pump and thereby provides real-time glucose measurements and trends. Calibration is required at least once every $12 \mathrm{~h}$. The pump is uploaded to transfer information to Medtronic CareLink therapy Management Software through the use of CONTOUR NEXT LINK glucose metre, which is also the uploading device.

'Suspend before low' is a SmartGuard function in the pump and suspends basal insulin infusion when sensor glucose is predicted to be below the set low limit in $30 \mathrm{~min}$. The pump suspends basal insulin infusion when two criteria are met: the sensor glucose is at or within $3.9 \mathrm{mmol} / \mathrm{L}(70 \mathrm{mg} / \mathrm{dL})$ above the set low limit and is predicted to be $1.1 \mathrm{mmol} / \mathrm{L}(20 \mathrm{mg} / \mathrm{dL})$ above the set low limit in $30 \mathrm{~min}$. The low limit is set for the entire study at $3.4 \mathrm{mmol} / \mathrm{L}$ and the pump would, therefore, suspend insulin infusion when the sensor glucose is 
$\leq 7.3 \mathrm{mmol} / \mathrm{L}(3.9+3.4)$ and predicted to be $4.5 \mathrm{mmol} / \mathrm{L}$ $(3.4+1.1)$ in $30 \mathrm{~min}$. If the alert before low is on, the patient will receive an alert when insulin delivery is suspended. Once the pump is suspended, the insulin infusion will resume after a maximum suspend period of $2 \mathrm{~h}$ or according to the autoresumption parameters if there is no patient interaction. Basal insulin will automatically resume if sensor glucose is above the low limit and trending upward, and insulin has been suspended for at least $30 \mathrm{~min}$. However, the infusion may be resumed earlier if the patient intervenes during the suspend time and overrides the suspend function. The autoresumption feature was not present in LGS wherein the pump had a full $2 \mathrm{~h}$ pump suspension in the absence of patient intervention and is novel to the predictive algorithm to reduce the potential of post suspend hyperglycaemia.

In the study, the low limit is set for Suspend before low feature. As previous in-clinic studies were evaluated with a suspend threshold of $80 \mathrm{mg} / \mathrm{dL}$ or $4.4 \mathrm{mmol} / \mathrm{L},{ }^{13}$ we maintained a similar threshold for the home study. As one of the purposes of this trial is to ensure safety of the system at an acceptable threshold which can also prevent hypoglycaemia, the low limit is set for the whole study to maintain uniformity in the intervention group and to enable comparisons. However, in real life, this low limit can be altered and the patient can have different low limits at various times of the day.

\section{Study protocol}

\section{VISITS 1 and 2: training}

As shown in figure 1, the first two visits are for training the participants: visit 1 for pump start and training and visit 2 for sensor training. Visits 1 and 2 are combined for participants previously competent with sensor use; however, for sensor naïve participants, visit 2 is undertaken at least 4 days after visit 1 . During these visits, participants are also trained to upload the pump at home, and are familiarised with sensor alerts and alarms. Sensor alerts and alarms are individualised for participants; every participant, however, has the alert on low turned on at $3.4 \mathrm{mmol} / \mathrm{L}$. Questionnaires are administered to participants and/or their parents at this visit. These include Clarke's hypoglycaemia awareness questionnaire, ${ }^{16}$ EQ-5D-Y and paediatric specific diabetes quality of life (PedsQL) questionnaires, ${ }^{17}$ Hypoglycaemia Fear Survey, ${ }^{18}$ and Pump Satisfaction questionnaire.

\section{Run-in-period}

The 2-week run-in period is designed to establish competent use of the system and thereby identifies participants who are not likely to comply with the protocol. All participants are required to use CGM for $>80 \%$ of the time during the run-in period and have to upload their pump for review by the investigators. Sensor naive participants are provided an additional week of CGM use to warrant adequate training and familiarisation. The sensor data is reviewed prior to randomisation to ensure a cohort that has and is prone to hypoglycaemia. The participant should have one or more sensor values $<3.5 \mathrm{mmol} / \mathrm{L}$ at any time during the period of CGM use, or one or more sensor values $<4.4 \mathrm{mmol} / \mathrm{L}$ on at least three different days. If the CGM is used successfully and the prerequisite criteria are met during the run-in period, participants return for visit 3 .

\section{VISIT 3: randomisation}

At this visit, measurement of HbA1c, height and weight are obtained, and incidence of moderate and severe hypoglycaemia is recorded from the patient and medical records. Moderate hypoglycaemia is defined as any episode of hypoglycaemia during which the child/adolescent was lethargic, disoriented, confused, and required third party assistance while severe hypoglycaemia is defined as an episode of loss of consciousness or seizure. ${ }^{19}$ Minimisation of variation in gender, age, HbAlc and hypoglycaemia unawareness score is undertaken at randomisation and performed using appropriate software (MinimPy).$^{20}$ Minimisation is a method of ensuring excellent balance between groups for known prognostic factors. ${ }^{21}$ Randomisations are undertaken by the delegated persons at Princess Margaret Hospital. Participants are randomised to standard therapy with SAPT alone (control group) or SAPT and Suspend before low (intervention group). Suspend on low function is turned off in the control group. Participants are instructed on ketone testing; before breakfast and pre-bed in both groups, and also at pump resumption after $2 \mathrm{~h}$ of suspend in the intervention group during the awake hours. They are also advised to test for ketones with glucose $>15 \mathrm{mmol} / \mathrm{L}$ or when unwell as part of routine diabetes care. ${ }^{22}$ They are instructed to contact the on-call paediatric endocrinologist or paediatrician for advice, if required. Ketone data are uploaded at the study visits four and five. Participants are encouraged to upload their pump fortnightly and are provided with a home record log book to document hypoglycaemia, ketosis and sensor or pump related events. Participants are advised to confirm all sensor alerts (on high, low and suspend) events with a capillary blood glucose value. The diabetes educator/study nurse will be in contact with participants regularly to provide any support needed and to ensure recording of adverse events.

\section{VISITS 4 and 5: follow-up visits}

At the follow-up visits at 3 months (visit 4) and 6 months (visit 5), the diabetes educator/study nurse ensures that all information has been uploaded from the pump and ketone metre. A measurement of HbAlc, height and weight will be obtained along with the record of moderate and severe hypoglycaemic episodes during this period. Participants and/or their parents' complete a quality of life questionnaire (EQ-5D-Y, PedsQL), Hypoglycaemia Fear Survey, Clarke's hypoglycaemia awareness questionnaire and Pump Satisfaction questionnaire during these visits. 


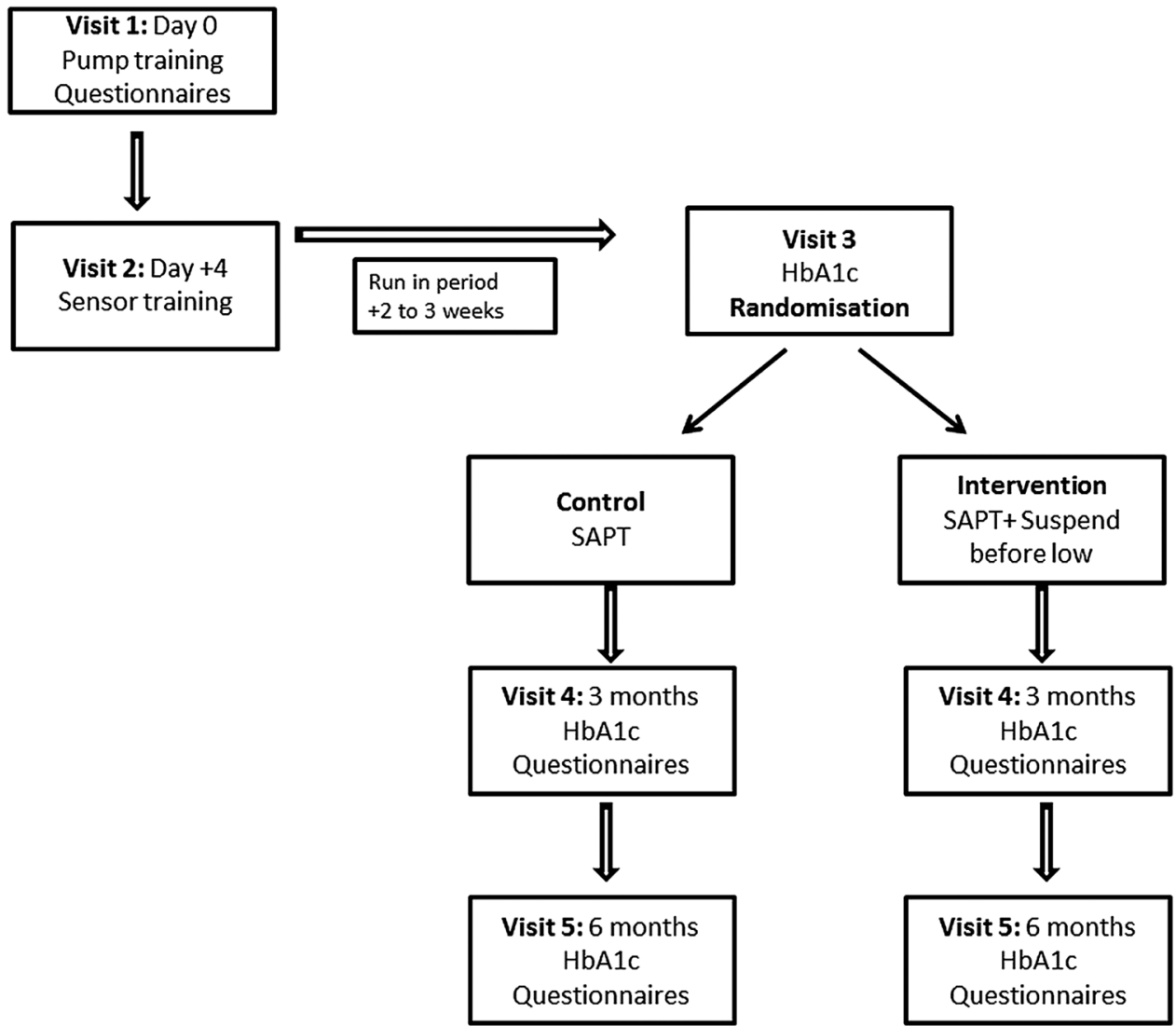

Figure 1 Study visits. Flow diagram represents the visits of the study. HbA1c, glycated haemoglobin; SAPT, sensor-augmented pump therapy.

\section{Hyperinsulinaemic hypoglycaemic clamps for participants} with impaired hypoglycaemia awareness

A score $\geq 4$ on Clarke's questionnaire indicates $\mathrm{IAH},{ }^{15}$ and participants with IAH and above 12 years of age are eligible for hyperinsulinaemic hypoglycaemic clamp studies which will determine the counter-regulatory responses to hypoglycaemia. The studies will be performed at baseline and after 6 months, irrespective of whether the participant is in the control or intervention group. The clamp procedure will involve infusing insulin intravenously at a constant rate of $80 \mathrm{mU} / \mathrm{m}^{2}$ per minute and plasma glucose targets will be achieved by adjusting the rate of infusion of a solution of $20 \%$ glucose in water. Prior to induction of hypoglycaemia, plasma glucose will be maintained in euglycaemia $(5-6 \mathrm{mmol} / \mathrm{L})$ for $60 \mathrm{~min}$ followed by gradual reduction over $30 \mathrm{~min}$ to a nadir of $2.8 \mathrm{mmol} / \mathrm{L}$. This controlled decline will be guided by plasma blood glucose measurements taken at 5 min intervals. The blood glucose concentration of $2.8 \mathrm{mmol} / \mathrm{L}$ will be maintained for $40 \mathrm{~min}$ before euglycaemia will be restored. For the duration of the clamp procedure, blood glucose will be measured using glucose oxidase technique with a bedside YSI analyser (Yellow Springs Instruments; Yellow Springs, Ohio, USA). Venous blood will be sampled during the euglycaemic and hypoglycaemic phase to determine plasma insulin, glucagon, epinephrine, norepinephrine, cortisol and growth hormone concentrations on both the study days.

\section{DATA MANAGEMENT AND MONITORING}

At consent, each participant will be given a unique identifying number based on their centre. The de-identified information with the patient's unique identifier code will be sent to the data manager in Perth and will be used as data input in the centralised database. Data will be stored in a secure office and password-protected computer files at Princess Margaret Hospital.

A data safety and monitoring board (DSMB) will scrutinise conduct of the study team and review the data arising from the study.

All adverse events defined as a clinical sign, symptom or condition that is causally related to the device implantation procedure, the presence of the device, or the performance of the device system will be recorded and evaluated by the local investigator. Serious adverse event is defined as one which is fatal or life-threatening or requires hospitalisation for diabetic ketoacidosis or severe hypoglycaemia. Adverse events and serious adverse events will be reported to the DSMB and to the ethics committee at each centre for their review.

\section{STATISTICAL ANALYSIS}

The analysis population will be the intention-to-treat population, which is defined as all patients who are randomised and have at least one visit after baseline. $\mathrm{p}$ Values $<0.05$ will be considered to be statistically 
significant and two-sided $\mathrm{p}$ values will be reported. The time spent hypoglycaemic or hyperglycaemic, and the continuous outcome measures will be analysed using a likelihood-based, mixed-effects model repeated measures approach. Rates of hypoglycaemia as well as incidence of moderate and severe hypoglycaemia will be analysed as unadjusted incidence rates based on the Poisson distribution. Incidence rates and incidence rate differences will be presented with their associated $95 \%$ CIs calculated as exact Poisson confidence limits. Number of ketosis events and other safety outcomes will be tabulated and presented as $\mathrm{n}$ and \%. The counterregulatory hormone responses to hypoglycaemia measured during the hyperinsulinaemic hypoglycaemic clamp study in participants with IAH will be presented with descriptive statistics.

\section{DISCUSSION}

This is the first multicentre randomised controlled home trial evaluating the performance of the 'Suspend before low' function in free-living conditions. Apart from the glycaemic data, the safety of the system can be monitored with ketones in both treatment groups. In addition, the 6-month study duration will provide us with the ability to evaluate the psychological outcomes by specifically addressing quality of life and fear of hypoglycaemia in both patients and their caregivers. The clamp data will also inform us of the counter-regulatory hormone responses in participants with IAH. An improvement in hormonal responses, if demonstrated, will enable us to use this system as a tool in this high-risk group to help restore awareness of hypoglycaemia.

SAPT with Suspend before low feature represents an important advancement in insulin delivery systems because of its potential to reduce hypoglycaemia. This study will be the first to quantify these effects in a randomised controlled trial of patients with type 1 diabetes who are predisposed to hypoglycaemia, and the results will provide a benchmark for further studies of automated insulin delivery systems.

\author{
Author affiliations \\ ${ }^{1}$ Department of Endocrinology and Diabetes, Princess Margaret Hospital for \\ Children, Perth, Western Australia, Australia \\ ${ }^{2}$ School of Paediatrics and Child Health, The University of Western Australia, \\ Perth, Western Australia, Australia \\ ${ }^{3}$ Telethon Kids Institute, The University of Western Australia, Perth, Western \\ Australia, Australia \\ ${ }^{4}$ Endocrinology and Diabetes Centre, Women's and Children's Hospital, \\ Adelaide, South Australia, Australia \\ ${ }^{5}$ Department of Endocrinology and Diabetes, John Hunter Children's Hospital, \\ Newcastle, New South Wales, Australia \\ ${ }^{6}$ Institute of Endocrinology and Diabetes, The Children's Hospital at Westmead \\ and The University of Sydney, Sydney, New South Wales, Australia \\ ${ }^{7}$ Department of Endocrinology and Diabetes, Royal Children's Hospital, \\ Melbourne, Victoria, Australia
}

Contributors TJ, ED, GA, FC, BK and JF conceived the study design; TL, NP, $\mathrm{HR}, \mathrm{MA}$ and JN contributed to the conception of the design. MA drafted the manuscript, and all authors reviewed the draft of the manuscript. All authors approved the final manuscript to be published.
Funding This study is funded by the Juvenile Diabetes Research Foundation funded Australian Type 1 diabetes Clinical Research Network. MA was funded by Channel 7 Telethon Research Fellowship 2014. The Jaeb Center for Health Research reviewed the protocol as part of the JDRF JDRF17-2011-664 Artificial Pancreas Consortium Network. Insulin pumps, transmitters, and glucose sensors were provided by Medtronic via an unrestricted grant. Additional pumps were also purchased through a grant from Princess Margaret Hospital foundation. Medtronic has no role in the design and conduct of the study; data collection, analysis and interpretation of the data; the preparation, review or approval of the manuscript; and decision to submit the manuscript for publication.

Competing interests This is an investigator-initiated trial. There are no competing interests.

Ethics approval Human Research Ethics Committee, Princess Margaret Hospital.

Provenance and peer review Not commissioned; externally peer reviewed.

Open Access This is an Open Access article distributed in accordance with the Creative Commons Attribution Non Commercial (CC BY-NC 4.0) license, which permits others to distribute, remix, adapt, build upon this work noncommercially, and license their derivative works on different terms, provided the original work is properly cited and the use is non-commercial. See: http:// creativecommons.org/licenses/by-nc/4.0/

\section{REFERENCES}

1. Barnard K, Thomas S, Royle $\mathrm{P}$, et al. Fear of hypoglycaemia in parents of young children with Type 1 diabetes: a systematic review. BMC Pediatr 2010;10:50

2. Choudhary $P$, Shin J, Wang Y, et al. Insulin pump therapy with automated insulin suspension in response to hypoglycaemia: reduction in nocturnal hypoglycaemia in those at greatest risk. Diabetes Care 2011;34:2023-5.

3. Garg S, Brazg RL, Bailey TS, et al. Reduction in duration of hypoglycaemia by automatic suspension of insulin delivery: the in-clinic ASPIRE study. Diabetes Technol Ther 2012;14:205-9.

4. Ly TT, Nicholas JA, Retterath A, et al. Effect of sensor-augmented insulin pump therapy and automated insulin suspension vs standard insulin pump therapy on hypoglycaemia in patients with Type 1 diabetes: a randomized clinical trial. JAMA 2013;310:1240-7.

5. Ly TT, Nicholas JA, Retterath A, et al. Analysis of glucose responses to automated insulin suspension with sensor-augmented pump therapy. Diabetes Care 2012;35:1462-5.

6. Bergenstal RM, Klonoff DC, Garg SK, et al. Threshold-based insulin-pump interruption for reduction of hypoglycaemia. $N$ Engl J Med 2013;369:224-32.

7. Ly TT, Gallego PH, Davis EA, et al. Impaired awareness of hypoglycaemia in a population-based sample of children and adolescents with Type 1 diabetes. Diabetes Care 2009;32:1802-6.

8. Cranston I, Lomas J, Maran A, et al. Restoration of hypoglycaemia awareness in patients with long-duration insulin-dependent diabetes. Lancet 1994;344:283-7.

9. Buckingham B, Chase HP, Dassau E, et al. Prevention of nocturnal hypoglycaemia using predictive alarm algorithms and insulin pump suspension. Diabetes Care 2010;33:1013-17.

10. Buckingham B, Cobry E, Clinton $\mathrm{P}$, et al. Preventing hypoglycaemia using predictive alarm algorithms and insulin pump suspension. Diabetes Technol Ther 2009; 11:93-7.

11. Buckingham BA, Cameron F, Calhoun $\mathrm{P}$, et al. Outpatient safety assessment of an in-home predictive low-glucose suspend system with Type 1 diabetes subjects at elevated risk of nocturnal hypoglycaemia. Diabetes Technol Ther 2013;15:622-7.

12. Maahs DM, Calhoun $P$, Buckingham BA, et al. A randomized trial of a home system to reduce nocturnal hypoglycaemia in type 1 diabetes. Diabetes Care 2014;37:1885-91.

13. Abraham M, de Bock M, Davey $R$, et al. Performance of a predictive algorithm in sensor-augmented pump therapy in the prevention of hypoglycaemia. Int J Pediatr Endocrinol 2015;2015:1.

14. Danne T, Tsioli C, Kordonouri O, et al. The PILGRIM Study: in silico modeling of a predictive low glucose management system and feasibility in youth with type 1 diabetes during exercise. Diabetes Technol Ther 2014;16:338-47.

15. Choudhary $\mathrm{P}$, Olsen BS, Conget I, et al. Hypoglycaemia prevention and user acceptance of an insulin pump system with predictive low glucose management. Diabetes Technol Ther Published Online First: 23 Feb 2016. doi:10.1089/dia.2015.0324. 
16. Clarke WL, Cox DJ, Gonder-Frederick LA, et al. Reduced awareness of hypoglycaemia in adults with IDDM. A prospective study of hypoglycaemic frequency and associated symptoms. Diabetes care 1995;18:517-22.

17. Varni JW, Curtis BH, Abetz LN, et al. Content validity of the PedsQL 3.2 Diabetes Module in newly diagnosed patients with Type 1 diabetes mellitus ages 8-45. Qual Life Res 2013;22:2169-81.

18. Cox DJ, Irvine A, Gonder-Frederick L, et al. Fear of hypoglycaemia: quantification, validation, and utilization. Diabetes Care 1987;10:617-21.
19. Ly TT, Maahs DM, Rewers A, et al. ISPAD Clinical Practice Consensus Guidelines 2014. Assessment and management of hypoglycaemia in children and adolescents with diabetes. Pediatric Diabetes 2014;15 20):180-92.

20. Saghaei M. An overview of randomization and minimization programs for randomized clinical trials. J Med Signals Sens 2011;1:55-61.

21. Altman DG, Bland JM. Treatment allocation by minimisation. BMJ 2005;330:843

22. Brink S, Laffel L, Likitmaskul S, et al. Sick day management in children and adolescents with diabetes. Pediatric Diabetes 2009;10 (Suppl 12):146-53. 